\title{
9
}

José Luis Káiser Moreiras*

Ángeles Bosch Jiménez**

\section{LA POLÍTICA COMERCIAL DE ESTADOS UNIDOS CON EL RESTO DEL MUNDO Los instrumentos de defensa comercial de EE UU en la era del presidente Trump}

Con la llegada del presidente Trump a la presidencia de EE UU, la política comercial norteamericana ha dado un giro de 180 grados. La seguridad nacional y la corrección del déficit comercial del país con sus principales socios son los dos motivos fundamentales que explican este cambio. Este giro se refleja en un cambio de actitud de EE UU en las negociaciones comerciales multilaterales, en sus relaciones bilaterales (sobre todo con China y, en menor medida, con la UE y Rusia) y en un aumento del recurso a las medidas unilaterales de defensa comercial, por parte de uno de los principales actores de la esfera internacional.

Palabras clave: OMC, proteccionismo, aranceles, salvaguardias, China, UE, NAFTA, KORUS, antidumping, instrumentos de defensa comercial, seguridad nacional, déficit comercial.

Clasificación JEL: F13, F52, K33.

\section{Introducción}

Para analizar la política comercial de un país debemos considerar las tres dimensiones de la misma: multilateral, bilateral y unilateral. Desde la llegada del presidente Trump a la Casa Blanca, las tres dimensiones han sufrido importantes variaciones en lo que venía siendo la posición tradicional de uno de los principales actores

\footnotetext{
* Director General de Política Comercial y Competitividad.

** Consejera Comercial de la Embajada de España en Washington

Versión de febrero de 2019.

DOI: https://doi.org/10.32796/bice.2019.3110.6781
}

del comercio mundial. Aunque muchos analistas consideran que estos cambios han hecho que la política de Trump sea impredecible, lo cierto es que está dando cumplimiento a lo que fue anunciando durante la campaña electoral previa a su elección como presidente de EE UU.

En política comercial, así como en otras políticas desarrolladas por la Administración americana, hay dos aspectos que adquieren una mayor importancia con respecto a Administraciones anteriores: la seguridad nacional y el crecimiento de la economía de EE UU.

Las consecuencias de la estrategia de seguridad nacional sobre la política comercial se reflejan en que EE UU no va a aceptar ninguna $\triangleright$ 
norma de comercio internacional que debilite internamente su economía. La consecuencia práctica de esta política es el incumplimiento de determinadas normas de comercio internacional. Asimismo, pone en el centro de la política comercial americana su conflicto con China y, en menor medida, con Rusia y la UE. Esta conexión de la seguridad nacional con la política comercial también implica la importancia clave que se otorga a la tecnología y a la protección de los derechos de propiedad intelectual e industrial. Es la justificación de las distintas medidas tomadas por EE UU bajo la sección 232 de la Trade Expansion Act de 1962 (seguridad nacional, medidas para acero, aluminio y automóviles) y bajo la sección 301 de la Trade Act de 1974 (medidas para proteger la propiedad industrial y en contra de la transferencia forzosa de tecnología).

El énfasis en el crecimiento económico de EE UU es la justificación utilizada para la reforma fiscal de diciembre de 2017, cuyos objetivos eran, en principio, la reducción de la carga fiscal de las familias de renta media, la mejora de la competitividad de las empresas norteamericanas y la repatriación de las rentas obtenidas fuera de EE UU.

\section{Política comercial multilateral}

Con respecto a la policía multilateral, las acciones de EE UU se centran en la OMC, en dos frentes: el Órgano de Apelación dentro del Mecanismo de Solución de Diferencias y la reforma/modernización de la OMC en su conjunto.

\subsection{Modernización de la OMC}

Con respecto a la modernización de la OMC, la posición de EE UU parte de un hecho objetivo: la organización ahora mismo es inoperativa con más de 162 miembros y teniendo que adoptar las decisiones por consenso. Hace tiempo que no avanza en sus negociaciones más importantes, sin ofrecer respuestas a los nuevos retos de la política comercial. Partiendo de esta premisa, la legitimidad de las respuestas que plantea la Administración americana suscita serias dudas. En resumen, lo que están proponiendo, y aplicando en la práctica, es la vulneración de las normas de comercio internacional que llevan décadas aplicándose. La Administración Trump contraargumenta diciendo que todas las Administraciones precedentes han puesto de manifiesto este problema y que, como utilizando los canales tradicionales y aplicando soluciones dentro de las normas no han obtenido resultados, no les ha quedado otra opción que no seguir el rule book.

La principal objeción de EE UU al funcionamiento de la OMC está en el cumplimiento de los objetivos de la Ronda Doha y en el Tratamiento Especial y Diferenciado. Con respecto a la Ronda Doha, los americanos consideran que es una cosa del pasado y que tras la X Conferencia Ministerial, celebrada en Nairobi en diciembre de 2015, se acordó que ya no existía un consenso sobre el mandato establecido en Doha en el año 2000. Por lo tanto, EE UU no se siente vinculado ni por los textos derivados de la Ronda Doha ni por el mandato establecido entonces, y considera que muchos países utilizan este mandato y estos textos como pretexto para estar exentos del cumplimiento de nuevas normas de comercio internacional. Solo unos pocos países, entre ellos EE UU, estarían obligados a cumplir dichas normas. Consideran que los pocos países que todavía defienden el mandato de Doha están perjudicando seriamente a la OMC porque impiden que avancen las negociaciones. 
Este hecho (referido a que solo unos pocos países cumplen toda la normativa internacional de comercio) es la principal preocupación de la Administración Trump. Las objeciones en este punto tienen una doble perspectiva. En primer lugar, cada país decide voluntariamente si se adhiere al Trato Especial y Diferenciado, y solo voluntariamente dejan de aplicárselo. En segundo lugar, no existen en la OMC unos criterios objetivos para clasificar a los países como PED o países de renta media. El resultado es que el tratamiento especial y diferenciado se lo aplican de igual manera países como China o Brasil y los países miembros menos avanzados. Por eso, este es uno de los puntos en los que EE UU está haciendo más hincapié.

Por último, pero no menos importante, EE UU quiere acabar con lo que considera un práctica habitual de países como China, que, a través del mecanismo de solución de disputas, consiguen evitar la aplicación de determinadas normas comerciales y han generalizado prácticas comerciales desleales y distorsionantes, en particular en materia de subvenciones. Esta posición de EE UU explica en gran medida su actitud hacia el Órgano de Apelación.

\section{2. Órgano de Apelación}

La principal objeción de EE UU al Órgano de Apelación (OA) es que a través de sus decisiones se vulnera el espíritu de las normas de comercio internacional adoptadas de común acuerdo por todos los países. Consideran que las decisiones del OA limitan sus derechos legítimos y vulneran la normativa OMC en casi todos los capítulos de la normativa internacional, pero especialmente en cuanto a subvenciones, derechos antidumping y derechos compensatorios; y salvaguardias. En cuanto a subvenciones, y una vez más en clara referencia a China, consideran que determinadas interpretaciones del OA suponen una limitación práctica para poder compensar subsidios proporcionados por empresas estatales. Con respecto a las salvaguardias, consideran que las decisiones del OA suponen la completa imposibilidad de aplicar el art. XIX del GATT, que establece esas medidas de salvaguardia (la sección 232 se refiere al art. XXI, medidas por seguridad nacional).

En la práctica, lo que está haciendo EE UU para promover los cambios deseados en el OA es vetar todos los nombramientos de nuevos jueces para el organismo. De esta manera, no se sustituye a los jueces que van cesando en sus funciones. A finales de 2019 el OA estará inoperativo completamente, al quedar un único juez para todos los casos que se planteen. La petición fundamental de EE UU en este ámbito es que se apliquen estrictamente las normas acordadas por lo Estados miembros de la OMC, tal y como están redactadas y que no se separen de la literalidad del texto en sus interpretaciones. Hay varios aspectos que preocupan a la Administración americana, pues los considera incumplimientos de las normas de funcionamiento del OA. Estos aspectos son los siguientes:

- La mayor parte de las decisiones del OA se toman fuera del plazo de 90 días establecido en las normas de funcionamiento, sin solicitud de extensión de plazo a las partes interesadas. Esta práctica tiene consecuencias importantes, pues en caso de autorizarse derechos compensatorios, estos se aplican con retrasos considerables, perdiendo su efectividad.

- La permanencia en el puesto de los jueces del OA. Los siete jueces del OA son elegidos para ejercer durante cuatro años, prorrogable por otros tantos años. $D$ 
Para EE UU, una vez superados dichos plazos, los jueces afectados dejan de ser miembros del OA y no pueden dictar resoluciones. La regla 15 de funcionamiento del órgano establece los motivos por los que se puede seguir considerando juez del OA a una persona cuyo mandato ha expirado. El argumento fundamental de EE UU en este punto es que solo el Órgano de Solución de Diferencias puede aplicar la regla 15, y la está aplicando el OA directamente, es decir, los propios jueces del OA se están autorizando a sí mismos prórrogas en su mandato.

- El OA incluye en sus decisiones opiniones y consejos sobre aspectos que no son necesarios para resolver la disputa planteada, «creando» normativa nueva con esas interpretaciones. Para EE UU, esta práctica se está generalizando y es perjudicial para el funcionamiento del mecanismo de solución de disputas.

- EE UU también critica que el OA interpreta ex novo, tanto legislación nacional de los Estados como hechos ya analizados en la primera fase del mecanismo de solución de disputas.

- Por último, EE UU critica que las decisiones del OA sean utilizadas como precedente en paneles posteriores. Lo consideran contrario a las normas OMC, ya que las normas de la OMC solo se pueden adoptar por consenso de todos sus miembros. Consideran que esta práctica vulnera su soberanía nacional.

\section{Política comercial bilateral}

El objetivo de política bilateral de la Administración Trump es conseguir acuerdos comerciales que beneficien a todos los americanos. Este objetivo ha tenido como consecuencia la revisión de varios acuerdos comerciales como el NAFTA y el acuerdo con Corea del Sur, la salida de EE UU del Acuerdo Transpacífico (TPP) y una política muy agresiva, de medidas unilaterales, con respecto a China y a la Unión Europea. En todas sus negociaciones bilaterales, el objetivo fundamental es el mismo: reducir el déficit comercial con sus socios.

\subsection{NAFTA/USMCA}

En este punto solo se analizarán las razones que han llevado a la Administración Trump a renegociar este acuerdo. Las nuevas condiciones acordadas con México y Canadá se analizan en otra parte de esta publicación.

Cuando el NAFTA entró en vigor el 1 de enero de 1994 todos los estudios del momento reflejaban que dicho acuerdo iba a suponer la creación de miles de empleos en EE UU (hasta 170.000 puestos de trabajo calculaban los más optimistas) y que el superávit norteamericano con México iba a aumentar exponencialmente. En contra de estas estimaciones, la Administración Trump considera que el NAFTA ha supuesto pérdida de empleos, cierre de factorías y la deslocalización de empresas fuera de EE UU. El déficit estadounidense con México ha pasado de 15.000 millones USD en 1995 a 71.000 millones USD en 2017.

Las razones de estas consecuencias negativas de la ratificación del NAFTA son claras para la Administración Trump. En primer lugar, a las empresas de EE UU el acuerdo les da la oportunidad de abrir factorías en México sin restricciones y pagando salarios más bajos. El capítulo de derechos laborales y sociales no está incluido en el acuerdo, sino que es un $\triangleright$ 
side agreement y no puede utilizarse en la solución de disputas.

El acuerdo tampoco ha servido para mejorar la productividad mexicana ni para reducir la brecha entre los salarios entre EE UU y México, objetivo que se perseguía con la firma del acuerdo. Además, se consideraba que la redacción inicial del NAFTA incentivaba la deslocalización de empresas fuera de EE UU.

Los objetivos de la Administración americana en la renegociación del NAFTA fueron dos: modernizar el acuerdo con disposiciones sobre los nuevos temas relacionados con el comercio internacional (comercio electrónico, ciberseguridad, propiedad intelectual) y reequilibrar los beneficios del NAFTA a favor de EE UU, es decir, reducir el déficit comercial que EE UU tiene con México y con Canadá.

\subsection{Acuerdo con Corea del Sur (KORUS)}

Para el acuerdo con Corea del Sur (KORUS) también consideraban que los resultados están muy por debajo de las expectativas y de las estimaciones iniciales. Como en otros casos, lo más importante es el dato de déficit comercial bilateral, el cual ha aumentado un $73 \%$ en los seis años en que el KORUS ha estado en vigor. Asimismo, la Administración Trump considera que Corea no estaba cumpliendo determinados compromisos en materia de derechos laborales, competencia y aduanas, asumidos en el ALC.

En octubre de 2017 se iniciaron las negociaciones para la modificación del acuerdo, y en septiembre de 2018 los presidentes de ambos países rubricaron el nuevo acuerdo. Las novedades principales afectan al sector del automóvil (reducción de aranceles para camiones, mayor reconocimiento de los estándares de seguridad americanos con intención de doblar las exportaciones de coches hacia Corea, reconocimiento de estándares para componentes y de estándares medioambientales norteamericanos), al farmacéutico (aumento de los reembolsos a medicamentos con origen en EE UU) y al ámbito aduanero (aceleración de los procesos de verificación).

\subsection{China}

La relación bilateral con China supone la principal preocupación de EE UU porque los dos factores que más inquietan a la Administración Trump, déficit comercial bilateral y seguridad nacional, alcanzan su máxima expresión con China.

Esta relación bilateral se encuentra condicionada por medidas unilaterales. Ahora las mencionaremos, como explicación de la reacción china, sin perjuicio de realizar un análisis más detallado de las mismas en la sección dedicada a la política comercial unilateral.

El 23 de marzo de 2018, el presidente de EE UU decidió imponer unilateralmente aranceles adicionales a la importación de determinados productos siderúrgicos y de aluminio, del $25 \%$ y $10 \%$, respectivamente, sobre la base de las recomendaciones de la investigación acerca de los efectos que tienen las importaciones de estos productos en la seguridad nacional de este país, bajo la sección 232 de la Trade Expansion Act de 1962, elaborado por el Departamento de Comercio estadounidense. El informe había sido encargado por el propio presidente Trump, en abril de 2017, por el efecto del exceso de capacidad en el sector siderúrgico mundial sobre el sector estadounidense y sus implicaciones para las necesidades de seguridad nacional de EE UU. 
En respuesta a estas medidas, el 2 de abril de 2018, China impuso aranceles adicionales del $15 \%$ a 120 productos de origen estadounidense, y del $25 \%$ para otras ocho mercancías, todo ello por valor de 3.000 millones de dólares. Sin embargo, China no respetó los plazos mínimos exigidos por la OMC ni otros aspectos procedimentales.

Por otra parte, y en virtud de investigación llevada a cabo bajo la sección 301 de la Trade Act de 1974, el Departamento de Comercio de EE UU determinó que los actos, las prácticas y las políticas de las autoridades chinas son discriminatorios y conllevan el robo de la propiedad intelectual estadounidense y la transferencia de tecnología obligatoria, entre otras cuestiones. En consecuencia, el presidente Trump hizo publicar, el 15 de junio de 2018, la primera lista de más de ochocientos productos procedentes de China, por un valor aproximado de 34.000 millones de dólares, a los que se les aplica un tipo arancelario adicional del $25 \%$ desde el 6 de julio de 2018. La respuesta de China no se hizo esperar y sus autoridades comerciales aprobaron, el 16 de junio de 2018, un arancel adicional del $25 \%$ sobre 545 productos importados de EE UU, cuya entrada en vigor fue el 6 de julio, ascendiendo el valor de las exportaciones afectadas por este paquete a 34.000 millones de dólares.

A continuación, el mismo día 6 de julio de 2018, y en represalia a la respuesta china, el país americano anunció una segunda lista de productos chinos que cubre, aproximadamente, 16.000 millones de dólares y casi trescientas líneas arancelarias, a las que se les aplica también un arancel adicional ad valorem del $25 \%$ desde el 23 de agosto de 2018 . En contestación a la segunda lista estadounidense, el mismo día de su entrada en vigor, el 23 de agosto, las autoridades chinas anunciaron un arancel adicional del $25 \%$ sobre 114 productos procedentes de EE UU, por valor total de 16.000 millones de euros.

El 17 de septiembre de 2018, The Office of United States Trade Representative (USTR) presentó una tercera lista de productos de origen chino a los que se les aplica un arancel adicional del 10\%, desde el 24 de septiembre, y que se elevaría al $25 \%$ en enero de 2019 . El volumen de importaciones afectadas se amplía mucho más, para esta lista, a 200.000 millones de dólares, equivalentes a más de 5.000 líneas arancelarias de productos. El 18 de septiembre de 2018, China respondió con una lista de 5.207 partidas, esta vez por un valor de 60.000 millones de dólares, según la cual 3.571 productos estadounidenses están sujetos a aranceles adicionales del $10 \%$, y otra lista de 1.636 productos a un arancel del $5 \%$. Estos aranceles entraron en vigor el 24 de septiembre. Como novedad, el anuncio de China mencionaba posibles represalias adicionales, pero en forma de medidas no arancelarias, en parte porque las exportaciones de EE UU a China no son tan abultadas como al sentido inverso. A finales de octubre, el presidente Trump anunció que EE UU impondría aranceles adicionales de entre el $10 \%$ y el $25 \%$ al resto de importaciones chinas (aproximadamente 257.000 millones de dólares) si no se lograba un acuerdo entre ambos países.

No obstante, en señal de buena voluntad para reanudar las negociaciones entre ambos países, en diciembre de 2018, EE UU suspendió la elevación del arancel del $10 \%$ al $25 \%$ para la tercera lista de productos de origen chino, durante noventa días a contar desde el comienzo de 2019. China, por su parte, también se comprometió a no aumentar los aranceles a los productos de automoción (equivalentes a 126.000 millones de dólares) $\triangleright$ 
estadounidenses, durante noventa días, desde enero de 2019.

El volumen total de comercio afectado por las medidas y respuestas de los dos países principales en el comercio internacional asciende a 360.000 millones de dólares. A pesar de las rondas de negociación entre ambos, tanto en Pekín (mayo y junio) como en Washington (mayo y agosto), con el fin de alcanzar una solución orientada a reducir el déficit comercial de EE UU y a resolver otras preocupaciones comerciales de este, como la protección de la propiedad intelectual o el apoyo público distorsionante a los sectores productivos en China, la situación no ha dado signos de remisión.

La última reunión se produjo en Pekín el 7 y el 8 de enero de 2019 sin avances significativos, pero suavizando el clima de «enfrentamiento». La siguiente reunión tuvo lugar en Washington el 30 y el 31 de enero, también al máximo nivel. Tampoco se alcanzaron resultados concretos. Ambos países tienen hasta el 30 de marzo para alcanzar un acuerdo, según la «tregua» fijada por el presidente Trump.

\subsection{Unión Europea}

Como ya se ha señalado, en marzo de 2018, el presidente Trump adoptó medidas por motivos de seguridad nacional bajo la sección 232 de la Trade Expansion Act de 1962, que implican la imposición de una tasa adicional del $10 \%$ a todas las importaciones de aluminio y de otra del $25 \%$ para el acero. La UE obtuvo una exención temporal, pero no se logró una solución definitiva, por lo que las medidas se aplican a la UE desde el 1 de junio de 2018. Durante ese tiempo, la Comisión Europea, además de intentar que la UE quedara excluida de manera definitiva de las sobretasas, diseñó en paralelo una estrategia de medidas complementarias en el marco de las normas OMC para defender los intereses de la UE.

Además, en mayo del mismo año, a instancia del presidente Trump, el Departamento de Comercio inició una investigación bajo la sección 232 sobre las implicaciones de seguridad nacional de las importaciones de automóviles y autopartes.

La tensión comercial sin precedentes que se habían generado a raíz de estas acciones en las relaciones transatlánticas propició en cierta medida el encuentro que se celebró el 25 de julio, entre los presidentes Trump y Juncker, y que permitió que se llegara a un acuerdo político, recogido en una declaración conjunta, que supone el inicio de negociaciones entre ambos bloques comerciales sobre una serie de elementos para los que, en principio, podría haber consenso:

- Trabajar para lograr un comercio bilateral de productos industriales (excluidos los automóviles) libre de aranceles, de barreras no arancelarias y de subsidios.

- Trabajar para reducir las barreras. Ambos presidentes expresaron su voluntad de que aumente el comercio de servicios, productos químicos, farmacéuticos, productos médicos y soja.

- Entablar un diálogo sobre normas para facilitar el comercio y reducir los obstáculos burocráticos y, en consecuencia, los costes.

- Fortalecer la cooperación estratégica en el ámbito de la energía.

Además, se acordó trabajar con socios afines para reformar la OMC y abordar las prácticas comerciales desleales, incluida la vulneración de derechos de propiedad intelectual, $\triangleright$ 
la transferencia forzada de tecnología, las subvenciones industriales, las distorsiones creadas por las empresas estatales y el exceso de capacidad. Para llevar adelante esta agenda conjunta, se decidió crear inmediatamente un Grupo de Trabajo Ejecutivo conjunto que examinase un ámbito de aplicación estrictamente definido por la declaración conjunta.

Es importante destacar que, mientras las negociaciones estén en curso, ambas partes han acordado que no se impondrán nuevos aranceles y que se reevaluarán los aranceles existentes. Sin embargo, por el momento, las medidas estadounidenses sobre el acero y el aluminio siguen vigentes. Por lo tanto, la UE también mantiene sus medidas de reequilibrio.

A principios de 2019 se reanudaron las conversaciones entre ambas partes. EE UU insiste en incluir los temas agrícolas dentro de la negociación. La UE reitera que no va a incluir agricultura en las negociaciones, dado que EE UU no está dispuesto a negociar el Jones Act, Buy America, contratación pública a nivel subfederal o Indicaciones Geográficas. Por su parte, EE UU quiere resultados tangibles que permitan reequilibrar su déficit comercial con la UE.

A mediados de enero de 2019, tanto la UE como EE UU han puesto en marcha el proceso negociador internamente (encaminado a obtener un mandato de negociación por parte de las autoridades competentes). Así, el pasado 11 de enero de 2019, el USTR publicó un Resumen de los Objetivos Específicos de Negociación (Summary of Specific Negotiating Objectives) para un acuerdo comercial con la UE. Dicha publicación da lugar a un periodo de treinta días, tras el cual el USTR podría empezar las negociaciones. Los objetivos reflejan una negociación amplia, con un alcance mucho mayor al recogido en la declaración conjunta Juncker-Trump de julio.
Destaca la inclusión de agricultura, la no exclusión de la reducción arancelaria para el sector del automóvil -aunque tampoco se incluye-, la voluntad de negociar sobre cuestiones sanitarias y fitosanitarias (SPS) - para la cual la UE tiene una postura claramente defensiva-, así como la inclusión de derechos de propiedad intelectual (DPI) -aunque sin protección de Indicaciones Geográficas (IG) - y de compras públicas, aunque excluyendo el nivel subfederal y manteniendo programas como el Buy America.

Por su parte, el pasado 18 de enero de 2019, la Comisión publicó las propuestas de mandato autorizando la apertura de negociaciones para un acuerdo con EE UU para la eliminación de aranceles en bienes industriales y en materia de evaluación de conformidad (así como dos anexos con las directrices de negociación).

Con respecto a la propuesta de mandato para eliminación arancelaria de bienes industriales, se reconoce el derecho de las partes a aplicar medidas de defensa comercial conformes a la OMC, a diferencia de la propuesta de EE UU, que no hace mención alguna a la OMC. Asimismo, la UE se protege frente a acciones unilaterales de EE UU (ya sea mediante el recurso a la sección 232, a la 301, o a cualquier otra similar) mediante la inclusión en el acuerdo de una cláusula que permita la suspensión unilateral de concesiones equivalentes $u$ obligaciones. Adicionalmente, establece que la Comisión suspenderá las negociaciones si EE UU adopta nuevas medidas contrarias a la UE, ya sea mediante el recurso a la sección 232 , a la 301 u otra similar, y fija como condición para la conclusión de las negociaciones que EE UU haya retirado previamente las medidas contra el acero y aluminio de la UE.

Con respecto a la propuesta de mandato sobre de evaluación de conformidad, se $\triangleright$ 
menciona (sin aclarar explícitamente) una posible relación con el actual Acuerdo de Reconocimiento Mutuo (ARM) y también se incluye una mención a una serie de requisitos que se deben cumplir. Sin embargo, no aclara si precisará de cambios legislativos y si se está pensando en una regulación de carácter horizontal o sectorial.

El resto de cooperación regulatoria no exige mandato, al estar amparada por la Decisión del Consejo, de 22 de junio de 1998, relativa a la celebración del Acuerdo sobre el Reconocimiento Mutuo entre la Comunidad Europea y Estados Unidos de América. En este sentido, se han identificado tres áreas más avanzadas: farmacéuticos, equipos médicos y ciberseguridad.

La negociación entre la UE y EE UU va a estar condicionada por importantes elementos en los próximos meses. A la posibilidad de que el presidente Trump imponga medidas sobre los automóviles europeos se une el calendario electoral. Las elecciones europeas de 26 de mayo de 2019 suponen que no habrá una nueva Comisión con plenos poderes hasta el mes de noviembre. $Y$ en esas semanas de noviembre la Administración americana empezará a concentrar recursos en la elecciones presidenciales de 2020. Asimismo, la comunicación por ambos lados, para ganarse a sus respectivas opiniones públicas tras lo ocurrido durante las negociaciones del TTIP, va a resultar clave.

\section{Los instrumentos de defensa comercial en la era Trump}

Son múltiples los mecanismos que los países tienen a su alcance para diseñar y articular su política comercial, pero sin duda alguna la forma y la frecuencia con la que se utilizan los instrumentos de defensa comercial nos pueden dar una idea clara de cuáles son los objetivos e intereses reales que persigue un país a la hora de definir sus relaciones comerciales con el resto de la comunidad internacional.

El concepto de instrumentos de defensa comercial tradicionalmente incluía las medidas antidumping, antisubvención, también llamadas medidas compensatorias, y las salvaguardias, instrumentos todos ellos regulados al amparo del GATT y en la actualidad sujetos a las disciplinas de los respectivos acuerdos de la OMC (Acuerdo Antidumping, Acuerdo de Subvenciones y Medidas Compensatorias y Acuerdo de Salvaguardias). Sin embargo, la propia evolución de la forma en que se ha venido desarrollando el comercio internacional y las acciones o comportamientos de algunos países han propiciado el establecimiento de nuevos mecanismos que, sin ser en puridad instrumentos de defensa comercial, tienen efectos similares y se sustentan, al menos en apariencia, bajo determinadas disposiciones de la OMC.

EE UU constituye un buen ejemplo de país que cuenta con una gran variedad de instrumentos que le permiten limitar sus importaciones bajo determinadas premisas. A lo largo de su historia ha ido incorporando a su legislación comercial distintos mecanismos para abordar de manera más idónea determinados tipos de comportamientos o acciones de terceros países y que afecten a sus intereses. Entre estos se podría mencionar la sección 232 de la Trade Expansion Act de 1962, que permite la introducción de limitaciones en las importaciones cuando las mismas pongan en riesgo la seguridad nacional del país.

Aunque algunos de estos mecanismos han tenido un uso limitado a lo largo del tiempo, en momentos de crisis o turbulencias económicas se han convertido en una herramienta a la que los mandatarios americanos han recurrido $D$ 
en mayor o menor medida, incluso en ocasiones con el fin de solventar problemas de naturaleza estrictamente interna. Esto es precisamente lo que ha ocurrido tras la llegada a la Casa Blanca del presidente Trump, siendo el objetivo del presente artículo analizar los cambios que se han producido en EE UU en este ámbito en los dos últimos años y, más concretamente, en la evolución de las medidas de defensa comercial tradicionales, así como en la mencionada sección 232, dada la especial repercusión que el uso de este instrumento ha tenido en el comercio internacional.

Desde el inicio de su campaña electoral, el candidato Trump dejó bien claro que, si llegaba a presidente de EE UU, defendería los intereses de los ciudadanos americanos, protegiendo sus productos, sus industrias y a sus trabajadores, y que haría uso de todos los mecanismos a su alcance para luchar contra las prácticas desleales que amenazaran la estabilidad y seguridad del país. Este mismo mensaje formó parte de su discurso inaugural como 45 presidente de EE UU, quedando además patente el cambio de rumbo que iba a tomar la política comercial del país. En tan solo dos años de legislatura, el presidente Trump ha pasado de las palabras a los hechos, y lo que podrían haber sido mensajes puramente electoralistas se han convertido en realidad, siendo precisamente el recurso a los instrumentos de defensa comercial uno de los mecanismos en los que ha centrado gran parte de su política comercial.

\subsection{Medidas antidumping $y$ antisubvención}

Este tipo de medidas, reguladas bajo sendos acuerdos de la OMC, están diseñadas para contrarrestar el daño ocasionado o la amenaza de daño que pueden causar a la producción doméstica de un país las importaciones de un producto realizadas en condiciones de dumping o que se han beneficiado de subvenciones específicas. Para imponer este tipo de medidas, que normalmente suelen adoptar la forma de aranceles a la importación adicionales, se requiere llevar a cabo una investigación en la que se debe determinar que se cumplen todos los requisitos que exigen los respectivos acuerdos de la OMC (existencia de dumping o subvención, daño material a la industria doméstica y vínculo causal entre el daño y el comportamiento desleal de las importaciones). Se inician normalmente a petición de la industria afectada, si bien también pueden iniciarse de oficio por parte de las autoridades del país importador.

En EE UU ambos tipos de medidas se regulan bajo la Ley Arancelaria de 1930, la cual ha sufrido numerosas modificaciones para ir adaptándose a los nuevos tiempos y también, cómo no, a los desarrollos normativos de índole internacional. Estas medidas, como no podía ser de otra forma, deben inspirarse y respetar los principios que emanan del Acuerdo Antidumping y del Acuerdo de Subvenciones y Medidas Compensatorias de la OMC. No obstante, el margen de maniobra que confieren los mismos dota a la normativa de cada país de sus propias particularidades, siendo precisamente la normativa de EE UU una de las más singulares, y sus procedimientos administrativos, de los más complejos de entre los miembros de la OMC.

En el caso de EE UU, este tipo de investigaciones se llevan a cabo en paralelo y de manera complementaria por parte del Departamento de Comercio, cuya labor se limita a la determinación del dumping y/o de la subvención, y la Internacional Trade Commission, $\triangleright$ 
competente en los aspectos relacionados con el daño. Además de la industria doméstica, los sindicatos americanos están también habilitados para presentar quejas antidumping y antisubvención.

Aunque en estos dos últimos años no se han llevado a cabo cambios significativos en la legislación básica por la que se regulan los procedimientos antidumping y antisubvención de EE UU — de hecho, la última reforma importante se produjo bajo la Administración Obama-, lo cierto es que la frecuencia con la que se han iniciado nuevos casos y la forma en la que se han venido desarrollando las correspondientes investigaciones ponen de manifiesto una deriva más proteccionista y una menor sensibilidad hacia los derechos de las partes acusadas de incurrir en prácticas de dumping o de beneficiarse de subvenciones ilícitas. En este sentido, se puede apuntar, entre otros, una mayor profusión en el tipo de información demandada, en muchos casos solicitando datos no pertinentes para la investigación; una aplicación restrictiva de los plazos a tener en cuenta en las distintas etapas del procedimiento; la no aceptación de datos facilitados por las partes por entender que no están completos; un uso abusivo de lo que se conoce como hechos disponibles adversos - situación que en muchos casos lleva a la adopción de derechos antidumping y/o antisubvención mucho más altos-; mayor celeridad en la adopción tanto de medidas provisionales como definitivas, etcétera.

Es también relevante a estos efectos el hecho de que, en marzo de 2017, se publicara una orden ejecutiva por la que se establece un plan estratégico destinado a combatir la elusión de derechos antidumping y antisubvención. De esta forma se logrará una aplicación más efectiva de estas medidas, al tiempo que se producirá con toda seguridad una mayor recaudación para las arcas públicas de derechos arancelarios hasta ahora no cobrados.

EE UU siempre ha destacado por tener un papel muy activo en lo que respecta a la adopción de medidas antidumping y antisubvención. Desde la creación de la OMC en 1995 hasta diciembre de 2017, último año completo del que existen registros en la secretaría de la OMC, EE UU ha iniciado 659 procedimientos antidumping y 219 antisubvención, imponiendo un total de 427 medidas antidumping y 122 antisubvención, circunstancia que lo convierte en el segundo miembro de la OMC, después de India, con el mayor número de medidas antidumping adoptadas en el mundo y en el primero en lo que respecta a las medidas antisubvención.

El mayor grado de intensidad en el recurso de este tipo de medidas ha ido variando sensiblemente a lo largo de los años, si bien es verdad que los periodos más convulsos económicamente hablando han venido coincidiendo con una mayor utilización de este tipo de instrumentos. En el caso de EE UU, y en lo que respecta a los procedimientos antidumping, el mayor número de inicios se produjo entre los años 1998 y 2004, con un promedio anual de 44 casos por año. A partir de ese momento se produce una importante bajada, con un repunte entre los años 2007 a 2009 y un nuevo incremento a partir de 2013 , que se ha visto exacerbado en 2017. En concreto, en 2017 se iniciaron 54 nuevos procedimientos antidumping, lo que supuso un incremento del $46 \%$ respecto a 2016, año en el que a su vez se detectó una bajada del $12 \%$ en el inicio de este tipo de investigaciones. La cifra del año 2017 es la más alta de los diez últimos años y, si se considera el periodo 1995-2017, solo estaría por debajo de la alcanzada en 2001, año en el que se iniciaron 77 nuevos procedimientos. Si nos atenemos al número de medidas antidumping $\triangleright$ 
adoptadas, el periodo más activo de EE UU fue el comprendido entre los años 1999 y 2002, con un promedio anual de 29 nuevas medidas, periodo que ha sido superado con creces en los años 2016 y 2017, en los que se han adoptado 35 y 33 nuevas medidas, respectivamente.

En lo que respecta a los procedimientos antisubvención, la situación ha sido parecida. Entre 1998 y 2001 se produjo un incremento en el número de casos abiertos, con una desaceleración importante y continua hasta 2013, año en que comienza a producirse un importante repunte, con 19 casos abiertos ese año, 18 en 2014, 23 en 2015, 16 en 2016 y 24 en 2017, siendo esta la mayor cifra desde 1995. En cuanto al número de medidas antisubvención impuestas, el periodo entre 1999 y 2001 fue uno de los más activos, volviendo a repuntar a partir de 2015, año en que se impusieron 10 nuevas medidas, que continuaron con 16 en 2016 y 11 en 2017.

Este importante uso de los procedimientos antidumping y antisubvención en 2017 se ha visto en cierta medida compensado por una menor actividad, sobre todo en lo que respecta a los inicios de las medidas antidumping, durante el primer semestre de 2018 (últimos datos de los que dispone la secretaría de la OMC). EI número de investigaciones antidumping iniciadas durante ese periodo ascendió a 23 , representando por tanto una disminución del 33\% respecto a la cifra alcanzada en el mismo periodo de 2017. Por el contrario, el número de medidas definitivas vigentes en los seis primeros meses de 2018 fue de 348 , en comparación con las 315 que existían en el primer semestre de 2017, lo que supone un aumento de algo más del $10 \%$. En lo que respecta a los casos antisubvención, el número de inicios se incrementó en un 13\% (17 en el primer semestre de 2018, en comparación con los 15 del mismo periodo del año anterior) y el de las medidas definitivas, en un 16\% (106 medidas en el primer semestre de 2018, comparado con las 96 en ese mismo periodo del año 2017).

La inmensa mayoría de los procedimientos iniciados y las medidas adoptadas en estos últimos años han tenido como objetivo primordial las exportaciones de la República Popular China, siendo el sector del acero el foco de muchas de ellas. Así, por ejemplo, de las investigaciones antidumping iniciadas en el primer semestre de 2018 el 39\% afectaron a China, y el $33 \%$ de las medidas definitivas iban dirigidas contra este país. En el caso de los procedimientos antisubvención, el porcentaje es mucho mayor: el $53 \%$ de los inicios correspondieron a China y el $46 \%$ de las medidas antisubvención se aplicaron a este país.

La UE ha sido también objeto de este tipo de medidas, algunas de las cuales han afectado especialmente a nuestro país. En concreto, en el año 2017 se iniciaron cinco procedimientos antidumping contra países miembros de la UE: fijaciones de acero de Italia, aceitunas negras de España, ácido cítrico de Bélgica, tubería mecánica de Alemania e Italia, y alambrón de Italia, España y Reino Unido, imponiéndose en todos los casos medidas definitivas en el transcurso de 2018. También se iniciaron dos investigaciones antisubvención: aceitunas negras de España y alambrón de Italia, habiéndose adoptado medidas en ambos casos en 2018. En 2018 se iniciaron cuatro nuevos procedimientos antidumping: tubos soldados y tubos soldados largos de Grecia, que en la actualidad se encuentran ya sujetos a medidas provisionales, y barriles de acero inoxidable de Alemania y cromato de estroncio de Austria y Francia, para los que de momento no se han impuesto medidas.

Sin duda alguna, de las investigaciones que han afectado a España las más notorias $\triangleright$ 
han sido las correspondientes a los procedimientos antidumping y antisubvención de aceitunas negras, no solo por el impacto económico que estas medidas están teniendo para el sector español de la aceituna negra (cuyas exportaciones al mercado americano han decrecido de manera espectacular al haberse adoptado aranceles suplementarios que van desde el $16,83 \%$ al $25,3 \%$ en el caso de las medidas antidumping, y del $7,5 \%$ al $27,02 \%$ en el procedimiento antisubvención), sino también por las implicaciones que este último procedimiento puede tener en el cuestionamiento indirecto que han realizado las autoridades americanas de los esquemas de ayudas de la política agrícola o de la políticas de desarrollo regional de la UE. Estos dos procedimientos han sido un claro ejemplo del nuevo cambio de política americana en lo que respecta al desarrollo de este tipo de investigaciones, habiéndose puesto en aplicación algunas de las nuevas prácticas comentadas con anterioridad, tales como la solicitud de información no apropiada a los efectos de la investigación, no aceptación de datos facilitados por las partes por entender que no estaban suficientemente claros 0 eran incompletos, o el recurso a los hechos disponibles adversos.

\subsection{Medidas de salvaguardia}

Las medidas de salvaguardia permiten a un país restringir temporalmente las importaciones de un producto si se determina que las mismas están creciendo de tal manera que han ocasionado o amenazan con causar un daño grave a la industria doméstica. Este tipo de medidas, que se incorporaron al artículo xix del GATT, se encuentran en la actualidad reguladas por el Acuerdo de Salvaguardias de la
OMC, y, en principio, el recurso a las mismas debería ser muy excepcional, dado el carácter disruptivo del comercio que tiene su aplicación. A diferencia de las medidas antidumping $\mathrm{o}$ antisubvención, las medidas de salvaguardia se aplican respecto a todos los países, siendo posible, no obstante, excluir de las mismas a aquellos países en vías de desarrollo si sus importaciones representan menos del $3 \%$ de las totales.

La especial naturaleza de estas medidas hace que su uso sea mucho menor que el de las medidas antidumping, o incluso antisubvención. Sin embargo, existen ciertos países, en general en desarrollo, en los que es más frecuente el recurso a este tipo de instrumento de defensa comercial.

En EE UU este tipo de medidas se encuentra regulado en la sección 201 de la Trade Act de 1974. Es la International Trade Commission (ITC) la encargada de llevar a cabo las correspondientes investigaciones, que pueden haber sido promovidas a instancias de la industria/ producción doméstica afectada por el incremento de las importaciones, los sindicatos, el representante de comercio de EE UU (USTR), el presidente de EE UU o incluso la propia ITC. Si la comisión determina afirmativamente que se está produciendo un daño o amenaza de daño importante, elaborará una recomendación al presidente, sugiriendo el tipo de medida y su duración para lograr remediar o prevenir el daño y facilitar así el reajuste de la industria doméstica. Es el presidente de EE UU el que tiene la última palabra respecto a su adopción. Una vez impuestas las medidas, son objeto de revisión periódica por parte de la ITC, que deberá informar al presidente de manera regular sobre su efecto en la producción doméstica. Cuando llegan a término, la ITC debe reportar al presidente $\mathrm{y}$ al Congreso americanos $D$ 
sobre su efectividad y la forma en que la industria doméstica se ha reajustado para poder hacer frente nuevamente a la competencia de las importaciones.

EE UU no es un gran usuario de este tipo de medidas, de hecho, desde la creación de la OMC hasta la fecha, tan solo ha impuesto ocho. Las dos últimas se adoptaron precisamente en 2018, y tras más de quince años sin que hubiera recurrido a este tipo de instrumento de defensa comercial. La primera de ellas se refiere a células fotovoltaicas para paneles solares, mientras que la segunda afecta a lavadoras de uso doméstico. El inicio de las correspondientes investigaciones se realizó en 2017, si bien las medidas, que en principio estarán en vigor por cuatro y tres años, respectivamente, se impusieron en 2018. Es importante señalar que ambas medidas han sido objeto de denuncia ante el Órgano de Soluciones de la OMC; el caso de las células fotovoltaicas, por parte de China y Corea del Sur; y el de lavadoras, por Corea del Sur.

Aunque, como se ha dicho anteriormente, EE UU no sea un actor relevante en lo que se refiere al recurso a este tipo de medidas, es bastante significativo que la llegada a la Casa Blanca del presidente Trump haya supuesto también una reactivación de un instrumento que prácticamente estaba en desuso en este país. Esta circunstancia no hace sino corroborar que los mensajes políticos de defensa a ultranza de la industria americana, a los que nos tenía tan acostumbrados el mandatario americano durante su campaña electoral, se están haciendo realidad.

Afortunadamente, en el transcurso del año 2018 no se ha iniciado ningún nuevo caso, pero, dado cómo está evolucionando la política comercial de EE UU en esos últimos tiempos, no sería descartable que la Administración norteamericana volviera a hacer uso de este tipo de medidas a corto plazo.

\subsection{Medidas de la sección 232 de la Trade Expansion Act de 1962}

Sin duda alguna, el cambio más notorio en lo que se refiere al uso de las medidas de defensa comercial o instrumentos asimilados por parte de la actual Administración americana ha sido precisamente el recurso a la sección 232 de la Ley de Expansión Comercial de 1962. En virtud de esta disposición, el presidente de EE UU puede limitar o restringir las importaciones de un producto cuando estas estén poniendo en riesgo la seguridad nacional. Para ello es necesario que el Departamento de Comercio americano lleve a cabo una investigación, la cual puede ser iniciada a solicitud de una parte interesada o a instancia del máximo responsable de cualquier Departamento o Agencia americana, incluido el propio Secretario de Comercio.

Esta sección fue introducida en la Ley de Comercio americana en el año 1962 en un momento especialmente delicado en el que EE UU se encontraba en plena Guerra Fría, de ahí que su concepción tuviera más que ver con elementos geoestratégicos que con los puramente comerciales. El contexto en que se diseñó nos puede dar una pista de la idea que subyacía en la mente de los legisladores americanos a la hora de introducir esta cláusula, estando sobre todo vinculada a cuestiones relativas a la defensa militar, y diseñada para activarla en situaciones de especial gravedad, de ahí que su uso debiera ser excepcional. Buena prueba de ello son las escasas ocasiones en las que se ha hecho uso de este tipo de medidas. Desde su creación hasta la fecha se han llevado a cabo 30 investigaciones. $\triangleright$ 
Entre 1980 y 2001, año en que por última vez se recurrió a este tipo de procedimiento, se ha utilizado esta cláusula en 14 ocasiones, habiéndose impuesto medidas en 7 de estos procedimientos: 4 para crudo y sus derivados (1982, 1989, 1994 y 1999); una para máquinas herramienta (1983); una para rodamientos antifricción (1988) y una para semiconductores (1993).

La llegada del presidente Trump a la Casa Blanca ha propiciado la reactivación de este instrumento comercial, siendo además muy significativo el hecho de que, a diferencia de lo que había ocurrido en ocasiones anteriores, la aplicación de este tipo de medidas haya generado una gran controversia, tanto en EE UU como a nivel internacional, habiéndose convertido en el detonante de lo que algunos economistas han catalogado como la primera guerra comercial del siglo xxI.

En concreto, en 2017, el Departamento de Comercio inició dos investigaciones para productos de acero y aluminio sobre la base de la sección 232, las cuales generaron además gran polémica interna al contar con importantes detractores por entender que era difícilmente argumentable que las importaciones de estos productos estuvieran poniendo en riesgo la seguridad nacional. El 8 de marzo de 2018 el presidente decidió imponer medidas en forma de una subida de aranceles del $25 \%$ para los productos del acero y del 10\% para el aluminio y sus derivados. En su opinión, las medidas estaban plenamente justificadas, ya que las importaciones de estos productos estaban afectando de manera muy importante a la industria siderúrgica y del aluminio de EE UU, haciéndolas comercialmente inviables, situación que podría acarrear consecuencias desastrosas tanto para el sector de defensa como para ciertas infraestructuras americanas vitales para la seguridad del país.
Estas medidas, que entraron en vigor el 22 de marzo, afectan a todos los países con la excepción de Australia, Corea del Sur, Argentina y Brasil. En el caso de Corea del Sur, su exclusión se produjo tras la renegociación de algunos de los elementos del acuerdo Corea del Sur-EE UU (KORUS), entre ellos la autolimitación por parte del país asiático del número de automóviles a exportar al mercado norteamericano. Argentina y Brasil, por su parte, quedaron también exceptuadas al aceptar limitar el nivel de sus exportaciones de acero y aluminio a EE UU. En un primer momento, Canadá, México y la UE quedaron temporalmente exentos. Con ello el presidente Trump pretendía, por una parte, propiciar que se terminara en un tiempo récord el proceso de modernización del Acuerdo NAFTA con Canadá y México y, por otra parte, sentar las bases para que la UE concediera un mejor acceso a productos norteamericanos y con ello resolver el alegado problema del déficit comercial que tiene EE UU con este área comercial y que viene siendo una de las grandes obsesiones de la actual Administración americana. Sin embargo, ante la imposibilidad a corto plazo de lograr esos objetivos, el presidente Trump decidió imponer las medidas a estos países a partir del 1 de junio de 2018.

La reacción de la comunidad internacional a la adopción de estas medidas no se hizo esperar, al considerar que difícilmente las mismas podrían encajar en las premisas de seguridad nacional que permite el artículo xxI del GATT, siendo más bien unas medidas de salvaguardia económica encubiertas, cuya imposición suponía una vulneración de las reglas de comercio internacional al no respetar el Acuerdo de Salvaguardias de la OMC ni el artículo XIX del GATT. Ello motivó que entre los meses de abril y agosto de 2018, China, India, Canadá, México, Noruega, Rusia, Suiza, Turquía y la $\triangleright$ 
UE decidieran recurrir estas medidas ante el Órgano de Solución de Diferencias de la OMC (OSD), el cual, de momento, ha aceptado la constitución de paneles, si bien todavía no se han iniciado los correspondientes trabajos. Al mismo tiempo, algunos de estos países decidieron optar por establecer medidas de reequilibrio, tal y como permite el Acuerdo de Salvaguardias, elevando los aranceles a determinados productos de EE UU por un valor equivalente al comercio afectado por las medidas americanas al acero y aluminio. La UE ha sido uno de ellos, adoptando medidas que se aplicarán en dos fases: la primera entró en vigor el 1 de junio, mientras que la segunda fase se producirá en tres años. Por su parte, EE UU ha considerado que las medidas de reequilibrio no estaban justificadas y suponen una violación de las reglas internacionales por no estar contemplada este posibilidad de compensación en el artículo xxI del GATT. En consecuencia, las ha recurrido ante el OSD, habiendo objetado (de momento) las medidas de reequilibrio adoptadas por Canadá, China, México, Rusia, Turquía y la UE.

Esta escalada de incremento de aranceles generalizada prácticamente no se había visto desde los años treinta, y es más que probable que vaya a tener un efecto muy negativo sobre los flujos de comercio a nivel internacional, con consecuencias totalmente impredecibles. Al mismo tiempo, la presentación de tantos casos ante el OSD no va a hacer sino bloquear, aún más si cabe, el trabajo de dicho órgano, que en los últimos años venía experimentando importantes retrasos, generándose, por tanto, una mayor incertidumbre sobre el resultado final de estos litigios y las implicaciones de los mismos.

La actividad de la actual Administración americana, en lo que se refiere al uso de la sección 232 , no ha terminado con la imposición de las medidas al acero y al aluminio, puesto que en 2018 se han abierto dos nuevos casos. El primero de ellos se inició en mayo y afecta a los automóviles y sus partes, mientras que el segundo se refiere a uranio y se abrió en julio.

Sin duda alguna, la investigación que tiene mayor trascendencia es la de automóviles, no solo porque supone un reto para el propio Departamento de Comercio, dada la complejidad y alcance de la misma, sino sobre todo por las repercusiones que tendría una eventual adopción de medidas. Si la imposición de aranceles para el acero y el aluminio ya generó controversia dentro de EE UU, esta investigación ha supuesto la movilización de grandes actores americanos, entre ellos los propios fabricantes de automóviles estadounidenses, e incluso congresistas, tanto demócratas como republicanos, que están en contra de que se adopte cualquier tipo de acción restrictiva. A ello habría que añadir la reacción de la comunidad internacional, que ya ha expresado sus dudas sobre la legitimidad que tendrían unas potenciales medidas y que con toda probabilidad reaccionaría imponiendo medidas de reequilibrio, en esta ocasión por valor muy superior a las del acero y el aluminio, y volviendo a plantear muy posiblemente nuevos litigios ante el OSD.

Por otra parte, no hay que negar que el mero hecho de que se haya iniciado esta investigación ha supuesto un revulsivo para que finalizara la modernización del acuerdo NAFTA, ahora rebautizado con las siglas USMCA, cuyas negociaciones concluyeron en agosto con México y en septiembre con Canadá. Estos países, que habrían aceptado la inclusión en este acuerdo de cláusulas especiales para el sector del automóvil en el supuesto de que EE UU terminara adoptando medidas sobre la base de la sección 232, confían al mismo tiempo en que la aprobación del USMCA, por parte del Congreso americano, lleve a la eliminación de los $\triangleright$ 
aranceles que les están aplicando para el acero y el aluminio. También se podría decir que, en cierta medida, esta nueva investigación basada en la sección 232 ha propiciado, a su vez, el lanzamiento de las negociaciones de un nuevo acuerdo comercial con Japón o, incluso, el acuerdo alcanzado en julio de 2018 entre el presidente Trump y el presidente de la Comisión Europea, Jean-Claude Juncker, y cuyo objetivo es lograr un mayor acercamiento comercial entre ambos bloques, el cual quedaría paralizado si EE UU decidiera imponer aranceles a los automóviles.

De momento se está a la espera de conocer los resultados de esta investigación, cuyo informe debe ser presentado al presidente a mediados de febrero de 2019, contando este con noventa días adicionales para tomar una decisión final.

\section{Conclusiones}

Es innegable que la entrada en la Casa Blanca de Donald Trump ha supuesto un cambio radical en la política comercial de EE UU, así como en el papel que desempeña en la actualidad como importante actor comercial en el mundo y en la forma en que se está relacionando con el resto de la comunidad internacional.

En primer lugar, ha reconfigurado la posición tradicional de EE UU a nivel multilateral. En términos OMC, esto supone un reto sin precedentes, pues la organización está al borde del colapso. En diciembre de 2019 dejará de estar operativo el Órgano de Apelación dentro del Mecanismo de Solución de Diferencias, que, por otro lado, es la gran diferencia entre el GATT y la OMC, y que dota a la OMC de una capacidad real de resolver las disputas comerciales e interpretar la normativa comercial internacional. Asimismo, tanto en sus negociaciones bilaterales como con sus medidas unilaterales, EE UU está vulnerando lo ya pactado a nivel multilateral.

Su política bilateral se basa fundamentalmente en la aplicación de medidas unilaterales que el resto de países debe aceptar si no quiere atenerse a las consecuencias de dichas medidas. Solo con China y con la UE, debido a su potencial comercial, se han prolongado realmente las negociaciones, pero siempre con medidas unilaterales encima de la mesa y bajo la amenaza de ampliarlas. En las próximas semanas podremos observar si esas negociaciones llegan a buen puerto y sirven para reducir la tensión comercial, o si han sido una excusa para retrasar la aplicación de nuevas medidas unilaterales y continuar con la escalada de tensión.

Si bien es cierto que en muchas ocasiones las acciones del mandatario americano han sido sorpresivas, no es menos cierto que, en materia comercial, y en particular en el área de la defensa comercial, sus promesas electorales las está poniendo en práctica y que el leitmotiv de esa política comercial es y seguirá siendo la de defender a ultranza los intereses americanos, su industria y sus trabajadores. Para ello no ha dudado en hacer uso de la amplia batería de instrumentos de defensa comercial con los que cuenta la legislación americana, y ello a pesar de que en algunas ocasiones dichas actuaciones hayan sido objeto de fuertes críticas internas, incluso por parte de importantes miembros de su propio partido.

En estos dos primeros años de su mandato se ha incrementado el ya de por sí uso importante que venía haciendo EE UU de los mecanismos de defensa comercial tradicionales, sobre todo en lo que respecta a las medidas $D$ 
antidumping y antisubvención, que han crecido de manera significativa, pero lo más relevante ha sido el recurso a las medidas de la sección 232 de la Trade Expansion Act de 1962 y la convulsión que han generado estas medidas en el comercio internacional. Nos encontramos en un momento especialmente delicado, tanto desde el punto de vista interno de EE UU como a nivel internacional, y en el que el grado de incertidumbre es bastante alto, pero todo apunta a que esta tendencia proteccionista del actual presidente de la Casa Blanca continuará, por lo que no habría que descartar nuevas medidas impactantes de aquí a que finalice su mandato.

Aunque todavía es pronto para valorar las consecuencias de esta política netamente proteccionista por la que ha optado la Administración Trump, lo cierto es que a corto plazo parecería que le ha dado ciertos réditos, sobre todo en el ámbito doméstico. Por de pronto, y de acuerdo con informaciones de la Administración americana, hay muchas industrias americanas que están satisfechas con ese mayor recurso a las medidas antidumping y antisubvención, ya que estarían permitiendo la contención de importaciones consideradas por ellos como desleales o ilícitas. Por otra parte, y aunque las medidas del acero y el aluminio cuentan con bastantes detractores y hayan desencadenado efectos colaterales en otros sectores, según fuentes oficiales, la situación de los fabricantes de estos productos habría mejorado al incrementar sus niveles de producción, pero sobre todo sus precios de venta, dejando atrás el potencial riesgo de desaparición que, según ellos, existía. Por último, y no menos importante, la Casa Blanca considera que haber tenido una actitud firme a la hora de imponer medidas para el acero y el aluminio o el mero hecho de haber iniciado una investigación de la sección 232 respecto al automóvil y la posibilidad de que puedan imponerse medidas en este sector podrían haber jugado un papel importante a la hora de reorientar los acuerdos NAFTA o KORUS, o incluso la formalización de posibles nuevos acuerdos con Japón y la UE. Es precisamente este planteamiento el más problemático de todos, ya que seguirá permitiendo al presidente Trump continuar su senda proteccionista y poner en jaque los fundamentos de las normas internacionales de comercio que hemos acordado entre todos y que tanto esfuerzo nos ha costado lograr. 